\title{
Genetic Ablation of Drosophila Phagocytes Reveals Their Contribution to Both Development and Resistance to Bacterial Infection
}

\author{
Arnaud Defaye $^{\mathrm{a}, \mathrm{d}}$ Iwan Evans $^{\mathrm{b}}$ Michèle Crozatier ${ }^{c}$ Will Wood ${ }^{\mathrm{b}}$ \\ Bruno Lemaitre $^{a}$ e François Leulier $^{a}$ \\ a Centre de Génétique Moléculaire, CNRS FRE 3144, Gif sur Yvette, France; b Department of Biology and Biochemistry, \\ University of Bath, Bath, UK; ${ }^{C}$ Centre de Biologie du Développement, UMR 5547 and IFR 109, CNRS and \\ Université Paul Sabatier, Toulouse III, Toulouse, and ${ }^{\mathrm{d} I n s t i t u t ~ d e ~ B i o l o g i e ~ d u ~ D e ́ v e l o p p e m e n t ~ d e ~ M a r s e i l l e-L u m i n y, ~}$ \\ CNRS UMR 6216, Université de la Méditerranée Aix-Marseille II, Marseille, France; ${ }^{\mathrm{G}}$ Global Health Institute, \\ Ecole Polytechnique Fédérale de Lausanne, Lausanne, Switzerland
}

\section{Key Words}

Haemocytes - Phagocytes - Drosophila $\cdot$ Invertebrate immunity

\begin{abstract}
Drosophila phagocytes participate in development and immune responses through their abilities to perform phagocytosis and/or secrete extra-cellular matrix components, antimicrobial peptides, clotting factors and signalling molecules. However, our knowledge of their functional impact on development and host resistance to infection is limited. To address this, we have used a genetic cell ablation strategy to generate Drosophila individuals lacking functional phagocytes. Our results highlight the essential contribution of phagocytes to embryonic development including central nervous system morphogenesis. Phagocytes also ensure optimal viability during post-embryonic development through immune functions. The use of phagocyte-depleted flies reveals the contribution of phagocytes in the resistance of Drosophila adults upon systemic infections with specific bacteria. Phagocytes were not involved in the expression of antimicrobial peptides by the fat body indicating a clear separation between cellular and humoral immune responses at this stage. Finally, we confirm that phagocytosis is a critical effector mechanism of the cellular arm by demonstrating
\end{abstract}

that phagocytosis contributes to resistance to infection with Staphylococcus aureus in adults. Our results highlight the power of this cell ablation strategy to reveal the contribution of phagocytes to specific biological processes. We now provide a blueprint of phagocyte importance during both development and innate immune responses in Drosophila.

Copyright $\odot 2009$ S. Karger AG, Basel

\section{Introduction}

In mammals, phagocytes of the blood cell lineage are essential components of the innate and adaptive immune systems and play a role in tissue homeostasis and embryogenesis [1]. Recent findings have revealed an important degree of conservation in the signalling pathways involved in the development and function of blood cells in vertebrates and various invertebrate phyla, including Drosophila melanogaster [2, 3]. Drosophila haemocytes, the insect blood cells, are now widely used to analyse in vivo cell migration and phagocytic processes $[4,5]$. However, our knowledge of their functional impact on development and host resistance to infection is limited.

As in vertebrates, Drosophila blood cells development occurs in two phases. A first haematopoietic wave takes place during embryogenesis when a population of hae-

Dr. François Leulier

Centre de Génétique Moléculaire

CNRS FRE 3144, Bâtiment 26

Avenue de la terrasse, FR-91190 Gif sur Yvette (France)

Tel. +33 16982 3200, Fax +33 16982 4386, E-Mail leulier@cgm.cnrs-gif.fr 
mocytes originate from the procephalic mesoderm, which will differentiate into either plasmatocytes or crystal cells [6-9]. Plasmatocytes, the Drosophila professional phagocytes, make up to $95 \%$ of all embryonic haemocytes. They are extremely long-lived and persist through the larval stages, some being still detected in adults [10]. These cells are highly migratory and ingest microorganisms as well as 'altered-self' particles such as apoptotic cell corpses by phagocy tosis $[4,5]$. Therefore, they are referred to as phagocytes hereafter. A second population of haemocytes, the crystal cells, comprises up to $5 \%$ of embryonic haemocytes, persisting until the onset of metamorphosis and contribute to larval melanization reactions $[11,12]$. A second wave of haematopoiesis occurs later, at the larval stages, in the lymph gland. The precursors of the lymph gland derive from the dorsal thoracic mesoderm and coalesce during embryogenesis to form the first paired lobes of the organ, which subsequently grow by cell proliferation during the first and second larval instars. By the late third instar larval stage, the lymph gland is the main site of haemocyte production in the animal, and the primary lobes can be divided into 3 structurally different zones: an outer cortical zone containing proliferating mature phagocytes and crystal cells, an inner medullary zone containing quiescent immature haemocytes, and a posterior signalling centre that acts as a niche [13-15]. The lymph gland is also the production site of a third type of haemocyte found only in larvae, the lamellocytes. Lamellocytes are large, adhesive cells devoted to the encapsulation of foreign bodies too large to be phagocytosed; these cells differentiate from the immature haemocyte pool only in response to specific conditions, such as parasitization of larvae by Hymenoptera [11]. Under normal conditions, the lymph gland phagocytes remain in the lymph gland throughout larval stages and enter the circulation only at the onset of metamorphosis and ingest doomed larval structures. At later stages, no haematopoietic organ is found. Consequently, all phagocytes present in the larval haemocoel are of embryonic origin while adult phagocytes, the only circulating cells at this stage, consist of a mixture of embryonic and lymph gland-derived phagocytes [10, 11].

Previous studies have revealed that Drosophila phagocytes participate in both development and immune responses $[4,16]$. Indeed, they contribute to central nervous system (CNS) morphogenesis during embryonic development through clearance of apoptotic corpses and deposition of extracellular matrix components [17-19]. They also execute at least four immune tasks: (1) they secrete antimicrobial peptides $[20,21]$ and clotting factors
$[22,23]$; (2) they engulf and digest micro-organisms through phagocytosis [5]; (3) they contribute to local and systemic tissue damage responses in larvae [24] and adults [25], and (4) they contribute, in larvae, to the systemic production of antimicrobial peptides by the fat body, the functional equivalent of the mammalian liver [26-28]. However, our knowledge of their functional impact on development and host resistance to infection is limited.

To test the functional importance of Drosophila phagocytes, we have generated animals depleted of phagocytes using a specific genetic cell ablation strategy and studied their development and capacity to resist infection. Our results strengthen the notion that Drosophila phagocytes contribute to development. They also reveal that phagocytes significantly contribute to host resistance to several bacterial infections in adults while being dispensable for humoral immune responses at this stage. Furthermore, we confirm that phagocytosis is an important effector mechanism of the cellular arm of Drosophila immune responses by demonstrating that phagocytosis contributes to host resistance to systemic Staphylococcus aureus infection in adults. Altogether, our analysis reveals the important contribution of Drosophila phagocytes to both development and immunity.

\section{Experimental Procedures}

\section{Fly Stocks}

Fly stocks and crosses were maintained on polenta-agar medium. To obtain Haemo ${ }^{\text {less }}$ embryos (embryos depleted of haemocytes), Bax was specifically expressed in all embryonic haemocytes by crossing UAS-bax/CyO-actin-GFP [29] males with serpent(hemo)-GAL4,UAS-GMA [30] virgin females at $29^{\circ} \mathrm{C}$. GMA encodes the actin-binding domain of moesin fused to GFP. Phago $^{\text {less }}$ larvae ( $h m$ l(delta)GAL4, UAS-eGFP/UAS-bax larvae) and adults were obtained following crossing during $24 \mathrm{~h}$ at $25^{\circ} \mathrm{C}$ of virgin flies $h \mathrm{ml}$ (delta)-GAL4,UAS-eGFP [31] with males UASbax/CyO-actin-GFP progenies were then switched to $29^{\circ} \mathrm{C}$. $\mathrm{Pa}$ rental control UAS-bax/CyO-actin-GFP and serpent(hemo)GAL4,UAS-GMA or sibling control hml(delta)-GAL4,UASeGFP/CyO-actin-GFP and serpent(hemo)-GAL4,UAS-GMA/ CyO-actin-GFP were used as appropriate. Conditional Phagoless animals were obtained by crossing virgin females $h m l$ (delta)GAL4,UAS-eGFP with males UAS-bax,Tub-GAL $80^{\text {ts }} / \mathrm{CyO}-a c$ tin-GFP. The activity of the GAL4 system was controlled by placing the progenies either at restrictive temperature $\left(29^{\circ} \mathrm{C}\right.$, GAL 80 off, GAL4 system on) or at permissive temperature $\left(18^{\circ} \mathrm{C}\right.$, GAL 80 on, GAL4 system off). Germ-free Phago ${ }^{\text {less }}$ animals were obtained by crossing germ-free virgin females $h m l($ delta)-GAL4, UAS-eGFP with germ-free males UAS-bax/CyO-actin-GFP on autoclaved standard medium. Germ-free parental stocks were generated by bleaching embryos and cultivating them on 
autoclaved polenta-agar medium. Relish ${ }^{E 20}\left(\operatorname{Rel}^{E 20}\right)$, spätzle $^{r m 7}$ $\left(s p z^{r m 7}\right), D f(3 R) D 605$ and $D f(3 R) T l-I$ fly strains were described previously $[32,33]$. The Tub-GAL $80^{\text {ts }}$ line was obtained from Bloomington Stock Center and recombined with UAS-bax/CyOactin-GFP flies. The UAS-GFP-IR was provided by the National Institute of Genetics (Mishima, Japan) stock centre. The fly lines UAS-Eater-IR\#1 (obtained from the Vienna RNAi stock centre, transformant ID 4301) targets the region 345-575 of the Eater ORF while the RNAi line UAS-Eater-IR\#2 (obtained from the National Institute of Genetics) targets the region 27-525. No off-targets of these RNAi constructs were detected using a web-based search tool (http://www.dkfz.de/signaling/tools.php). 4- to 7-dayold adults flies were used in all experiments.

\section{Viability Studies}

Stage 14-17 serpent (hemo)-GAL4,UAS-GMA/UAS-bax (Hae$m o^{\text {less }}$ ) or serpent (hemo)-GAL4,UAS-GMA (wild type) embryos laid and raised at $29^{\circ} \mathrm{C}$ on apple juice agar plates supplemented with yeast paste were counted and separated under a fluorescent dissection microscope and allowed to develop further at $29^{\circ} \mathrm{C}$. Viability was scored by counting the number of larvae that hatched after $24 \mathrm{~h}$ and again at $48 \mathrm{~h}$. Post-embryonic viability was assayed by comparing the number of Phago $^{\text {less }}$ adults [ $h m l$ (delta)GAL4,UAS-eGFP/UAS-bax] and wild-type siblings [ $h m l$ (delta)GAL4,UAS-eGFP/CyO-actin-GFP] derived from crosses between hml(delta)-GAL4,UAS-eGFP females and UAS-bax/CyO-actinGFP males. Crosses were performed at $25^{\circ} \mathrm{C}$ and transferred at $29^{\circ} \mathrm{C}$ after $24 \mathrm{~h}$. A $100 \%$ being arbitrary attributed to the number of emerging wild-type sibling controls.

\section{Haemocyte Observation and Counts}

$h \mathrm{ml}$ (delta)-GAL4,UAS-eGFP/CyO-actin-GFP (wild type) and hml(delta)-GAL4,UAS-eGFP/UAS-bax (Phago $\left.{ }^{\text {less }}\right)$ larvae or adults were anaesthetized and viewed under epifluorescent illumination (excitation filter $480 \mathrm{~nm}$, dichroic filter $505 \mathrm{~nm}$, emission filter $510 \mathrm{~nm}$ ) with a Leica MZFLIII (Heerburg, Switzerland) dissecting microscope. Images were recorded with a charge-coupled device camera (Sony). For haemolymph observations, wild-type and Phago ${ }^{\text {less }}$ larvae were rinsed in sterile PBS, opened using forceps at the level of the posterior segment. Haemolymph samples were observed directly under a microscope (Leica DMRB) with either differential interference contrast or epifluorescent illumination. Circulating cells in wild-type and Phago ${ }^{\text {less }}$ larvae were counted per optical field using a haemocytometer under differential interference contrast illumination. Circulating cells expressing weak or strong GFP were counted in the same optical field under epifluorescent illumination. Acridine orange $(\mathrm{AO})$ staining was used to reveal apoptotic corpses and acidic cell compartments. Haemocytes collected from wild-type and Phago ${ }^{\text {less }}$ larvae were incubated with $10 \mu \mathrm{l}$ of $1.6 \mu \mathrm{M}$ AO in PBS and observed under epifluorescent illumination.

\section{Antibody Staining}

serpent (hemo)-GAL4,UAS-GMA/UAS-bax (Haemo ${ }^{\text {less }}$ ) and serpent (hemo)-GAL4,UAS-GMA/CyO-actin-GFP (wild type) embryos laid and raised at $29^{\circ} \mathrm{C}$ were fixed and co-immunostained. Rabbit anti-GFP (1/1,000; Abcam Inc.) was used to detect the presence or absence of the fluorescent balancer in combination with mouse anti-fascin (sn7c concentrate, diluted 1/100; Developmental Studies Hybridoma Bank, DSHB) to detect embry- onic haemocytes (I. Evans and W. Wood, unpubl. data); BP102 mouse anti-CNS (supernatant diluted 1/100; DSHB) or mouse anti-fasciclin 2 (1D4 supernatant diluted 1/5; DSHB) were used in combination with anti-GFP to show CNS morphology. Primary antibodies were detected with FITC-conjugated goat anti-mouse (1/200; Jackson ImmunoResearch Laboratories) and Alexa Fluor 594-conjugated goat anti-rabbit (1/200; Molecular Probes). Immunostained embryos were visualized using a Leica LSM510 confocal microscope, and $\mathrm{Z}$-stack projections were assembled using ImageJ.

Lymph glands from $h m l$ (delta)-GAL4,UAS-eGFP/CyO-actinGFP (wild type) animals were dissected, fixed for $5 \mathrm{~min}$ in $4 \%$ glutaraldehyde in $0.1 \mathrm{M}$ sodium phosphate buffer ( $\mathrm{pH} 7.3$ ), stained with primary rabbit polyclonal anti-GFP (1/1,000; Torrey Pines Biolabs) and TOPRO3 (Molecular Probes) to reveal nuclei, mounted in Vectashield medium (Vector Laboratories, Burlingame, Calif., US) and observed under confocal microscopy on a Zeiss LSM 510 microscope (Zeiss, Oberkochen, Germany).

\section{Bacterial Strains and Infection Experiments}

Injuries were performed by pricking 4 - to 7 -day-old adult females in the lateral thoracic region with a thin needle either clean (washed and rinsed in $70 \%$ ethanol) or previously dipped into a concentrated pellet of the following bacteria. Flies were incubated at the indicated temperatures. Erwinia carotovora carotovora 15 (Ecc15): optical density (OD) at $600 \mathrm{~nm}=170,29^{\circ} \mathrm{C}$; Escherichia coli: OD 160, $25^{\circ} \mathrm{C}$; Enterococcus faecalis: OD 5, $25^{\circ} \mathrm{C}$; Bacillus subtilis: OD $35,29^{\circ} \mathrm{C}$; Staphylococcus saprophyticus: OD $30,25^{\circ} \mathrm{C}$; Streptococcus agalactiae: OD 10, $25^{\circ} \mathrm{C}$; Enterobacter cloacae: $\mathrm{OD}$ $100,29^{\circ} \mathrm{C}$; Salmonella enterica serovar typhimurium: OD 200, $29^{\circ} \mathrm{C}$; S. aureus: OD $10,25^{\circ} \mathrm{C}$, or a mixture of Micrococcus luteus and E. coli: $\mathrm{OD} 100,25^{\circ} \mathrm{C}$. Peptidoglycan (PGN) preparations and injections were performed as previously described [32]. Survival analyses were performed on 20 adult females and repeated at least 3 times.

\section{Quantitative Real-Time PCR}

SYBR Green quantitative real-time PCR analysis was performed as previously described [32]. Primer information can be obtained upon request. The amount of mRNA detected was normalized to control rp49 mRNA values. Normalized data were used to quantify the relative levels of a given mRNA according to cycling threshold analysis $(\Delta \mathrm{Ct})$. Relative $\Delta \mathrm{Ct} \mathrm{t}^{\text {gene }} / \Delta \mathrm{Ct}^{\mathrm{rp} 49}$ ratios of unchallenged wild-type controls were anchored in 1 to indicate fold induction. Graphs represent the mean and SD of relative ratios detected in 3 biological repetitions of a pool of 10 females.

\section{Results}

\section{Drosophila Haemocytes Are Required for Embryonic Development}

In order to reveal the functional importance of Drosophila haemocytes, we have used a genetic system to perform cell-specific ablation by promoting targeted ectopic cell death. This relies on the use of the yeast UAS/GAL4 binary system [34] to direct the expression of the murine 

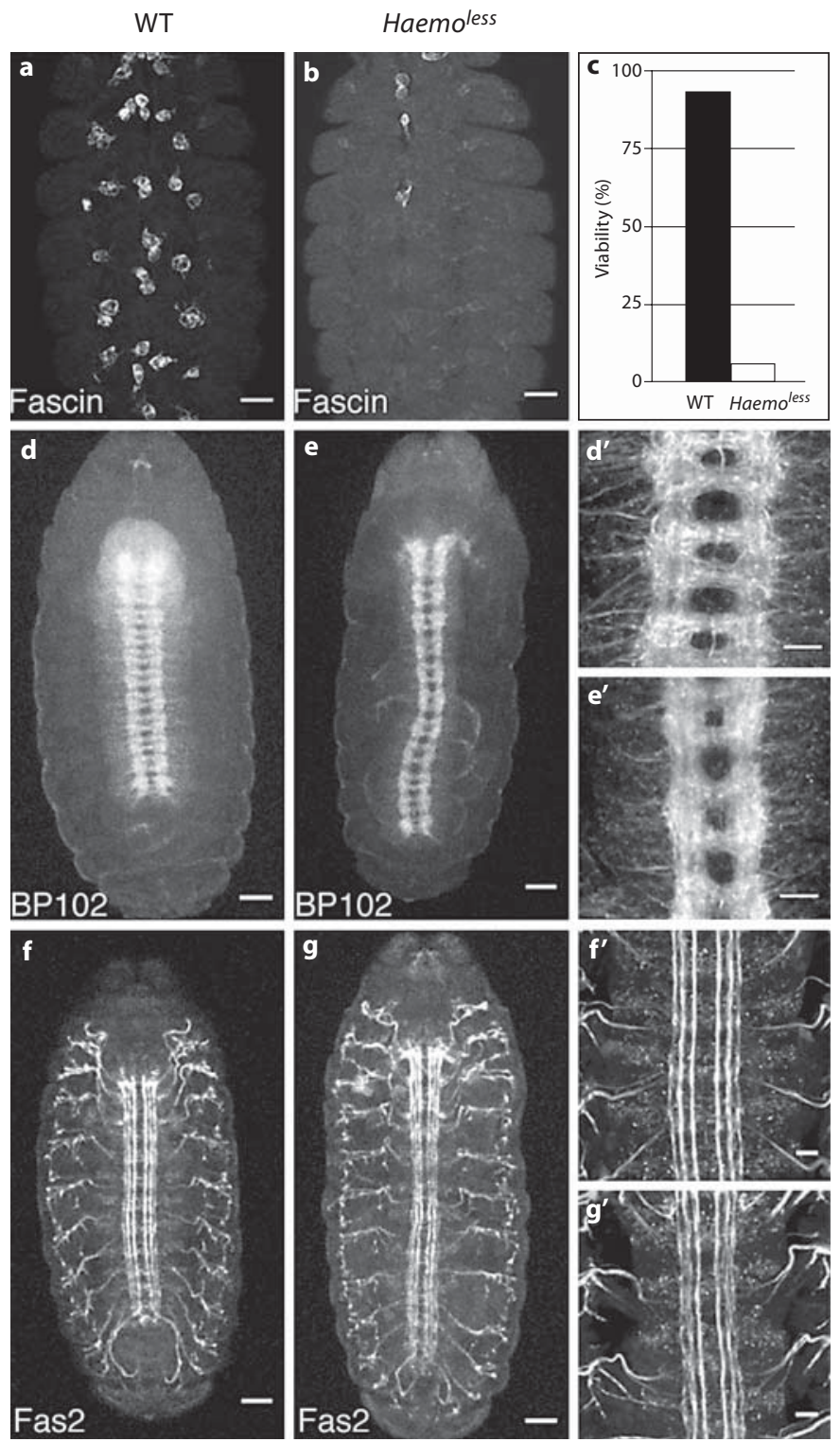

Fig. 1. Drosophila haemocytes contribute to embryonic development. a, b Anti-fascin staining of stage 14 wild-type (WT) and Haemo $^{\text {less }}$ embryos reveals near complete ablation of embryonic haemocytes in Haemo ${ }^{\text {less }}$ embryos. Anti-fascin staining allows visualization of haemocytes. Scale bars $=20 \mu \mathrm{m}$. c Developmental viability of wild-type (WT, $\mathrm{n}=150)$ versus Haemo ${ }^{\text {less }}(\mathrm{n}=162)$ embryos expressed as the percentage of first instar larvae emerging from laid eggs. d-g BP102 staining (d, e) and fasciclin 2 (Fas2; $\mathbf{f}, \mathbf{g})$ staining of stage 17 wild-type and Haemo $^{\text {less }}$ embryos reveal CNS condensation failure but otherwise grossly normal CNS architecture in Haemo ${ }^{\text {less }}$ embryos. Scale bars $=30 \mu \mathrm{m}$. BP102 stains all CNS axons while fasciclin 2 stains longitudinal CNS axon tracts. $\mathbf{d}^{\prime}-\mathbf{g}^{\prime}$ Enlargements of panels $\mathbf{d}-\mathbf{g}$. The anterior is up in all images. Genotypes: wild type: serpent (hemo)-GAL4,UAS-GMA/ CyO-actin-GFP (a, d, f) or serpent(hemo)-GAL4,UAS-GMA (c); Haemo $^{\text {less }}$ : serpent(hemo)-GAL4,UAS-GMA/UAS-bax (b, e, g).

Drosophila Phagocytes in Development and Immunity bax gene. Bax is a potent pro-apoptotic member of the Bcl-2 family, which promotes mitochondrial permeabilization [35] and efficiently triggers Drosophila apoptotic pathways [29]. Embryonic haemocyte identity is specified by the expression of the GATA factor serpent. serpent is required for specification of the haemocyte primordium within the head mesoderm at an early embryonic stage and, later, for adequate modification of the gene expression profile during haemocyte maturation [7]. To promote the ectopic death of most embryonic haemocytes, we drove the expression of a UAS-bax transgene using the serpent (hemo)-GAL4 driver. This driver is specific to all embryonic blood cell types and expresses from early haemocyte differentiation onwards [30]. serpent(hemo)-GAL4-mediated Bax expression led to almost complete deletion of embryonic haemocytes at stage 14 (fig. 1a, b). The lack of haemocytes was clearly associated with a strong lethality with only $6 \%$ of $\mathrm{Haemo}^{\text {less }}$ embryos $(\mathrm{n}=162)$ reaching the first instar larval stage (fig. 1c). This strongly suggests that haemocytes are essential for normal embryonic development.

Previous studies had revealed that embryonic haemocytes promote CNS morphogenesis through clearance of apoptotic corpses and deposition of extracellular matrix components [17-19]. Therefore, we wondered if CNS morphogenesis was affected in Haemo ${ }^{\text {less }}$ embryos. During Drosophila embryogenesis, the ventral nerve cord (VNC) axons establish a precise pattern reiterated in each segment forming two longitudinal tracts that run the length of the embryo on either side of the midline, with a subset of these axons crossing the midline (fig. $1 \mathrm{~d}, \mathrm{~d}^{\prime}$ ). During stage 16 and 17 of embryogenesis, the VNC undergoes a condensation phase along the antero-posterior axis. We observed that the VNC of Haemo ${ }^{\text {less }}$ embryos at stage 17 was longer and narrower than that of wild-type embryos (compare fig. 1e, g with fig. 1d, f). This indicates a failure of CNS condensation in these animals. Although CNS architecture was normal in Haemo ${ }^{\text {less }}$ embryos, the precise ladder-like axonal scaffold seen in wild-type embryos was disrupted leading to a rounding of the spaces between anterior and posterior commissures (fig. le, $\mathrm{e}^{\prime}$ ). Despite differences in axon scaffold shape, no inappropriate midline crossing of axons was detected (compare fig. If with fig. 1g). In wild-type animals, 3 major tracts of fasciclin-2-positive axons are observed near the dorsal surface of the CNS on either side of the midline (fig. 1f, $\left.f^{\prime}\right)$. Three tracts of fasciclin-2-positive axons were also observed in Haemo ${ }^{\text {less }}$ embryos (fig. 1g, g'). Although these tracts were relatively normal, they did show very mild defasciculation in some segments (fig. $1 g^{\prime}$ ). Alto- 
gether, these results indicate that embryonic haemocytes are required for CNS morphogenesis.

\section{Drosophila Phagocytes Are Essential for Optimal}

Viability during Post-Embryonic Development

Using the same strategy, we next addressed the functional impact of phagocytes during post-embryonic development. To overcome the essential requirement of haemocytes during embryonic development, we generated flies expressing Bax under the control of the $h m l$ (delta)-GAL4 driver. This transgene exclusively drives strong GAL4 expression in mature phagocytes from early first instar larval stage to adulthood [31] (fig. 2a, b, c', $\mathrm{g}, \mathrm{i}, \mathrm{k}$, and data not shown). Of note, this driver expresses in both pools of phagocytes those of embryonic origin, which are sessile or in circulation in larvae (fig. 2a, $c^{\prime}$ ), and those in the cortical zone of the lymph gland, which will populate adults (fig. $2 \mathrm{~b}$ ). $\mathrm{hml}$ (delta)-GAL4-mediated expression of Bax led to a massive disruption of the lymph gland during early larval stages (data not shown). The haemolymph of $h m l$ (delta)GAL4, UAS-eGFP/UASbax larvae $\left(\right.$ Phago $\left.^{\text {less }}\right)$ showed a large amount of apoptotic corpses, revealed by AO staining (fig. $2 \mathrm{f}, \mathrm{f}^{\prime}$ ) and a significantly reduced amount of circulating cells with an average of $74 \%$ decrease compared with wild-type larvae

Fig. 2. Drosophila phagocytes are partially redundant for postembryonic development. a Wild-type (asterisk) and Phago ${ }^{\text {less }}$ (arrows) wandering third instar larvae observed under epifluorescence illumination. Sessile and circulating larval phagocytes express the eGFP under the control of the $h m l$ (delta)-GAL4 driver. b Dissected lymph gland from $h m l(d e l t a)-G A L 4, U A S-e G F P /$ CyO-actin-GFP (wild type) third instar larvae stained with antiGFP and TOPRO3 to reveal nuclei. $h m l$ (delta)-GAL4-positive phagocytes are observed in the cortical zone of the primary lobes. c-f, $\mathbf{c}^{\prime}-\mathbf{f}^{\prime}$ Circulating haemocytes from wild-type (c, $\mathbf{c}^{\prime}$ and $\left.\mathbf{e}, \mathbf{e}^{\prime}\right)$ or Phago ${ }^{\text {less }}\left(\mathbf{d}, \mathbf{d}^{\prime}\right.$ and $\left.\mathbf{f}, \mathbf{f}^{\prime}\right)$ larval haemolymph, non-treated $\left(\mathbf{c}, \mathbf{c}^{\prime}\right.$ and $\mathbf{d}, \mathbf{d}^{\prime}$ ) or stained with AO to reveal apoptotic corpses and acidic cellular compartments (e, $\mathbf{e}^{\prime}$ and $\left.\mathbf{f}, \mathbf{f}^{\prime}\right)$, observed under differential interference contrast (c-f) or epifluorescent illumination $\left(\mathbf{c}^{\prime}-\mathbf{f}^{\prime}\right) . \times 40$ objective $(\mathbf{c}, \mathbf{d}) ; \times 20$ objective $(\mathbf{e}, \mathbf{f}) ; 400 \mathrm{~ms}$ exposure $\left(\mathbf{c}^{\prime}\right) ; 800 \mathrm{~ms}$ exposure $\left(\mathbf{d}^{\prime}\right) ; 200 \mathrm{~ms}$ exposure $\left(\mathbf{e}^{\prime}, \mathbf{f}^{\prime}\right)$. g Quantifications of circulating cells per optical field showing weak (as in $\mathbf{d}^{\prime}$ ) or strong (as in $\mathbf{c}^{\prime}$ ) fluorescent signal under epifluorescence illumination in wild-type (WT) and Phago ${ }^{\text {less }}$ larvae expressed as the percentage of circulating cell number observed in wild-type larvae under differential interference contrast (DIC) illumination. $\mathbf{h}$ Relative developmental viability of Phago ${ }^{\text {less }}$ individuals raised under conventional $(\mathrm{CR}, \mathrm{n}=295)$ or germ-free $(\mathrm{GF}, \mathrm{n}=$ 191) conditions at $29^{\circ} \mathrm{C}$. Shown are the percentage of emerging adults compared with their respective wild-type siblings [ $\mathrm{hml}$ -
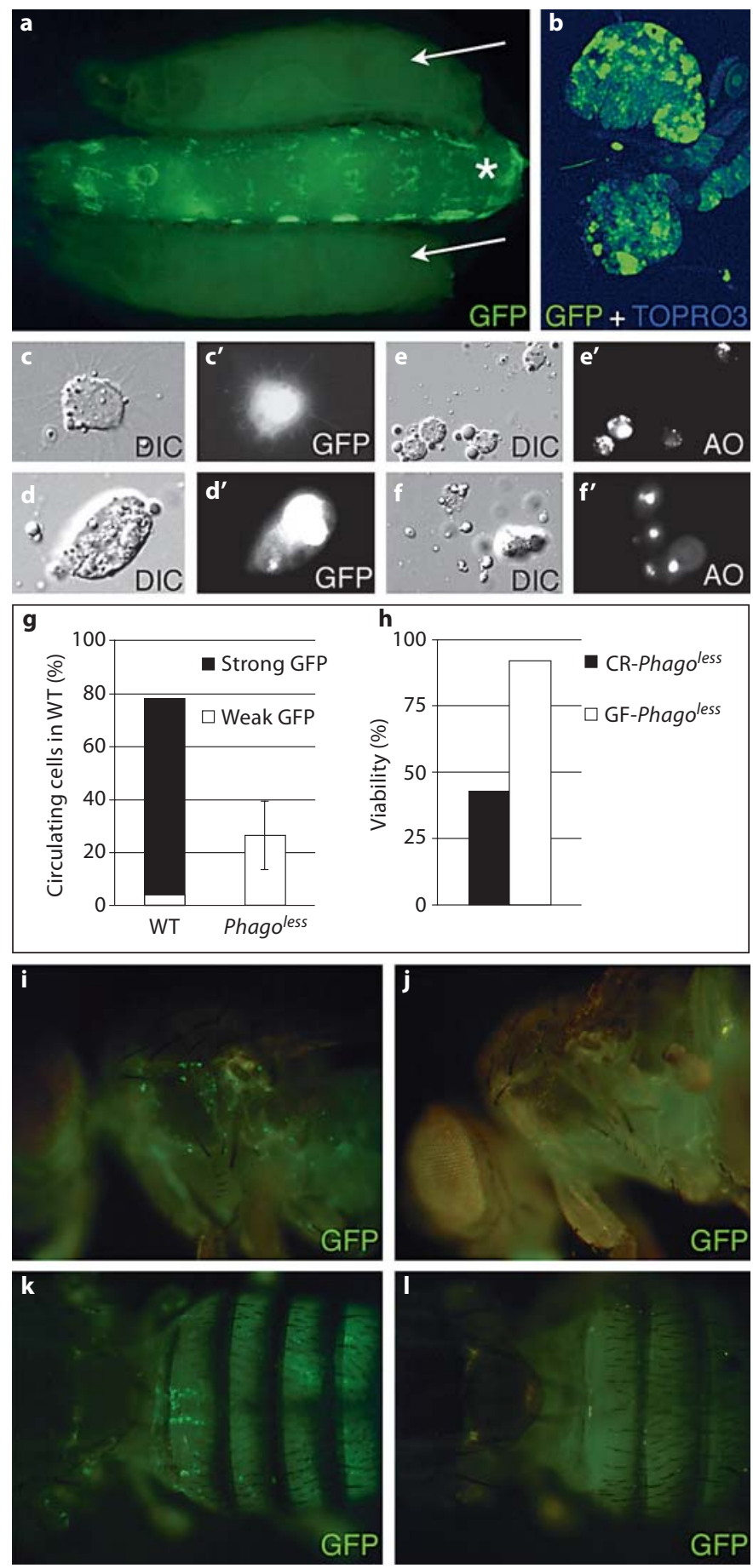

(delta)-GAL4,UAS-eGFP/CyO-actin-GFP] anchored to $100 \%$. i-I Phago ${ }^{\text {less }}$ adults (j, I) and sibling controls (i, k) observed under epifluorescence illumination. Lateral view of the thoracic region $(\mathbf{i}, \mathbf{j})$; dorsal view of the abdomen $(\mathbf{k}, \mathbf{I})$. Note the accumulation of sessile GFP-positive phagocytes on adult tissues (i) or circulating GFP-positive phagocytes along the dorsal vessel (k). Genotypes: wild type: $h m l($ delta)-GAL4,UAS-eGFP/CyO-actin-GFP; Phago $^{\text {less }}$ : hml(delta)-GAL4,UAS-eGFP/UAS-bax; siblings controls: $h m l($ delta)-GAL4,UAS-eGFP/CyO-actin-GFP. 
(fig. $2 \mathrm{~g}$ ). The remaining circulating cells appeared enlarged with an unusual morphology and lacked filipodia in sharp contrast to wild-type phagocytes (compare fig. $2 c$, e with fig. $2 d, f$ ). These enlarged cells weakly expressed the UAS- $g f p$ transgene driven by the $h m l$ (delta)GAL4 driver (fig. $2 \mathrm{~d}^{\prime}$, exposition time twice that of fig. $\left.2 c^{\prime}\right)$ and were unable to phagocytose AO-positive apoptotic corpses (fig. $2 \mathrm{f}^{\prime}$ ). In addition, phagosomal acidification, a key feature of phagosome maturation, which can be revealed by $\mathrm{AO}$ staining, did not occur in contrast to wild-type circulating phagocytes (fig. 2e', $\mathrm{f}^{\prime}$ ). Although the remaining circulating cells observed in Phago ${ }^{\text {less }}$ larvae may correspond to the previously identified population of undifferentiated circulating cells that are normally present in Drosophila larvae [11], we hypothesize that they rather correspond to a population of non-functional phagocytes at an early step of the apoptotic process or to abnormal lamellocytes released during the early rupture of the lymph gland. Nevertheless, these results indicate that efficient phagocyte ablation occurred during larval stages of developing Phago ${ }^{\text {less }}$ animals.

Remarkably, Phago ${ }^{\text {less }}$ adults were recovered and no hml(delta)-GAL4-positive circulating or sessile cells were observed through the cuticle (fig. $2 \mathrm{j}, \mathrm{l}$ ) or attached to dissected tissues (data not shown). In collected haemolymph, no circulating cell could be identified indicating that complete depletion of adult phagocytes occurred (data not shown). The recovery of viable and fertile adults deprived of phagocytes suggests that they are partially redundant for post-embryonic development to proceed. Indeed, they presented no obvious morphological defects apart from small melanotic masses found occasionally in the abdomen. However, phagocyte depletion still had a profound impact on developmental viability since, compared with wild-type siblings, $43 \%$ of the Phagoless animals reached adulthood when conventionally reared $(\mathrm{n}=$ 295; fig. 2h). This reduced viability may stem from a developmental function of phagocytes as suggested by previous reports showing that during metamorphosis, phagocytes ingest doomed larval tissues, in particular muscle cells [11]. Alternatively, phagocytes may ensure essential immune functions during larval stages to protect the host against opportunistic infections. To challenge these hypotheses, we generated germ-free Phago ${ }^{\text {less }}$ individuals and reared them under sterile condition. In this context, the developmental lethality of Phago ${ }^{\text {less }}$ animals was almost completely rescued to wild-type levels ( $\mathrm{n}=191$; fig. $2 \mathrm{~h}$ ). Altogether, these results indicate that Drosophila phagocytes are dispensable for normal postembryonic development to proceed. However they are required for optimal viability during post-embryonic development probably through immune functions protecting the host to infection by environmental micro-organisms.

\section{Reduced Resistance of Phago ${ }^{\text {less }}$ Adults to Bacterial Infections}

Using Phago ${ }^{\text {less }}$ adults, we next addressed the functional impact of Drosophila phagocytes in adult fly resistance to bacterial infection. To this end, we used an established model of systemic bacterial infection by septic injury, which consists of wounding the adult thoracic cuticle with a needle previously dipped into a concentrated bacterial solution. In the past, this infection model had been successfully used to reveal the essential contribution of Toll and immune deficiency (Imd) signalling pathways in host resistance to systemic bacterial infection. Upon infection by different classes of micro-organisms, Toll and Imd pathways control the expression of particular sets of immune effector genes, including genes encoding antimicrobial peptides in the fat body [16]. As a consequence, Toll pathway mutants such as those affecting the $s p z$ gene present a striking susceptibility to systemic infection by several Gram-positive cocci while Imd pathway mutants such as those affecting the Rel gene show a dramatic susceptibility to systemic infection by several Gram-negative rods [16]. Therefore, we compared the resistance of Phago ${ }^{\text {less }}$ flies to wild-type flies and $s p z$ or Rel mutants after septic injury using various bacterial strains.

Phagocyte-depleted adults showed a slight reduction in resistance to clean wounding compared with wildtype flies or Rel and $s p z$ mutants (fig. 3b) while they behaved as wild-type, $s p z$ and Rel flies in absence of challenge during the course of our survival analysis (fig. 3a).

(For figure see next page.)

Fig. 3. Reduced resistance of Phago ${ }^{\text {less }}$ adults to bacterial infections. Survival analysis of Phago ${ }^{\text {less }}$, wild-type siblings, wild-type parental control, conditional wild-type, conditional Phago less, Relish $^{E 20}$ and $s p z^{r m 7}$ flies in absence of injury (a), upon clean injury (b) or Ecc15 (c), E. coli (d), E. faecalis (e), B. subtilis (f), S. saprophyticus (g), S. agalactiae (h), S. typhimurium (i) or S. aureus (j, k) septic injury. Twenty adult females were used per experiment, and a representative experiment out of a minimum of 3 repetitions is shown. Genotypes: Phago ${ }^{\text {less }}$ : hml(delta)-GAL4,UASeGFP/UAS-bax; wild-type sibling: $h m l$ (delta)-GAL4,UAS-eGFP/ CyO-actin-GFP; parental wild type: UAS-bax/CyO-actin-GFP; conditional wild type: UAS-bax,Tub-GAL80 8 ts $/$ CyO-actin-GFP; conditional Phago ${ }^{\text {less }}$ : hml(delta)-GAL4,UAS-eGFP/UAS-bax, Tub-GAL80 $0^{\text {ts }}$. 

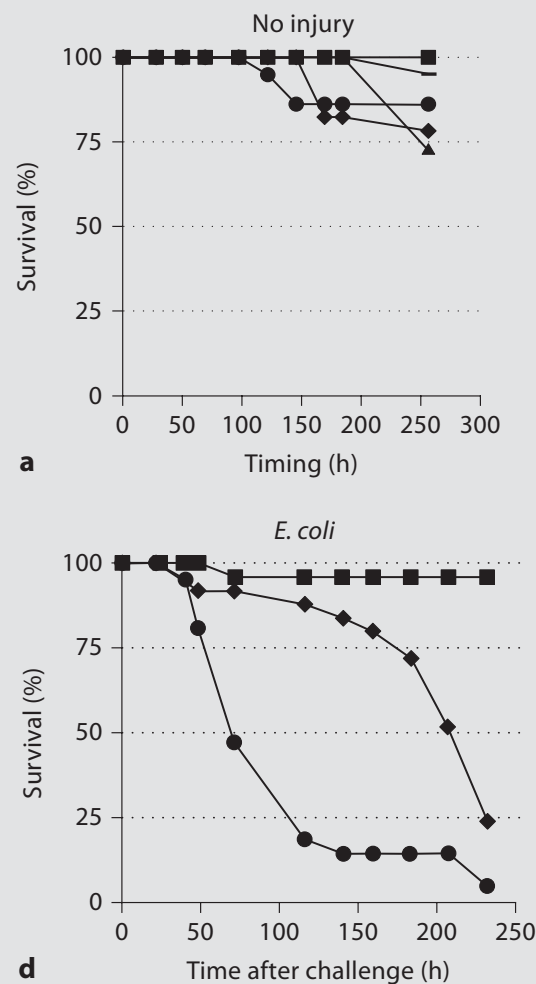

d

S. saprophyticus

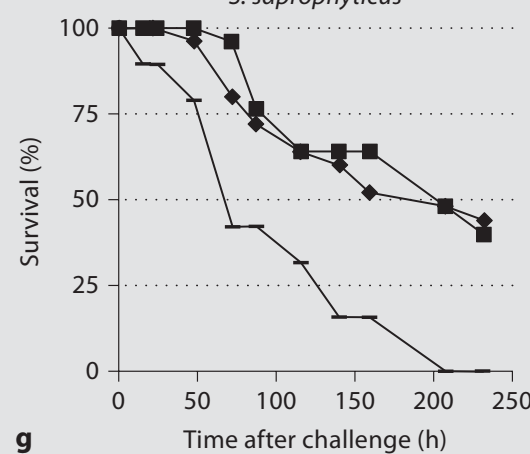

g

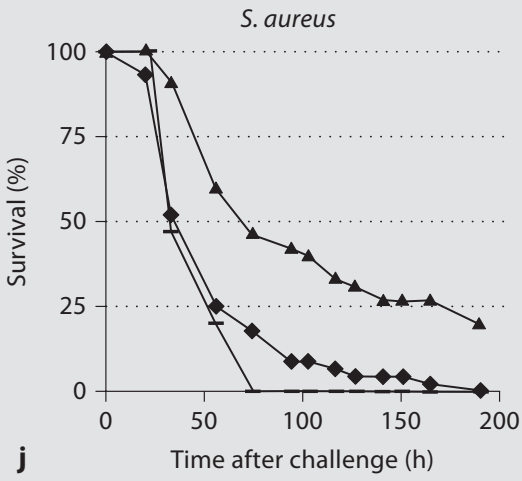

Clean injury

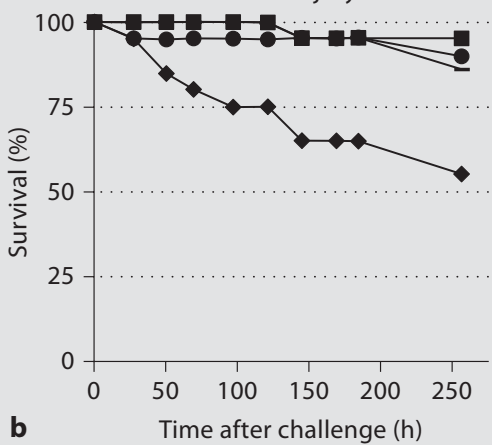

E. faecalis

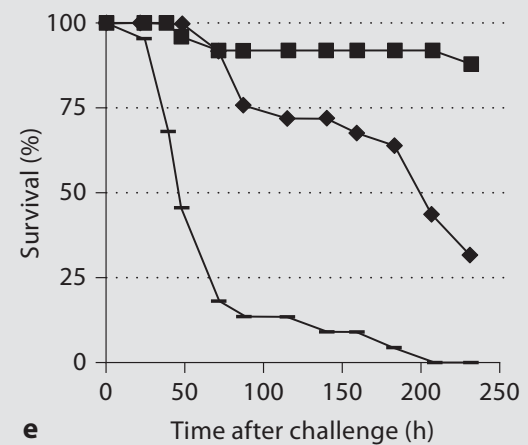

S. agalactiae

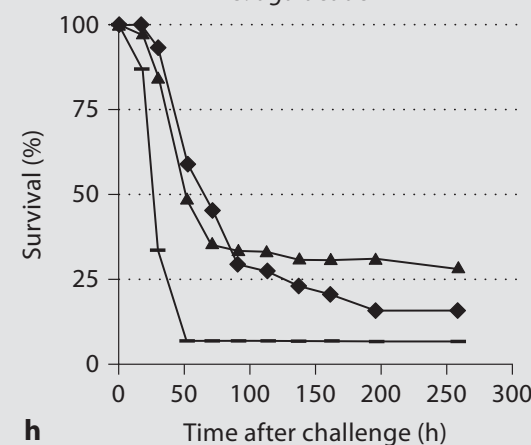

S. aureus

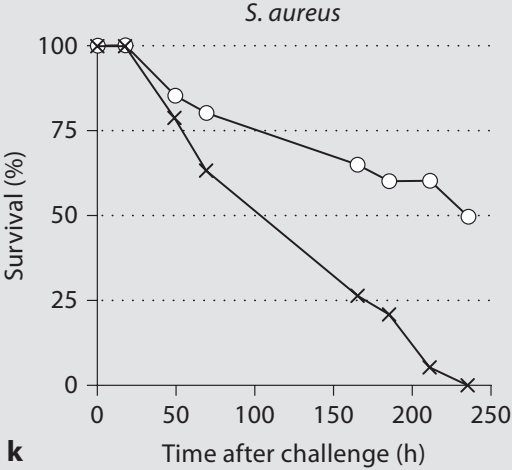

Ecc15

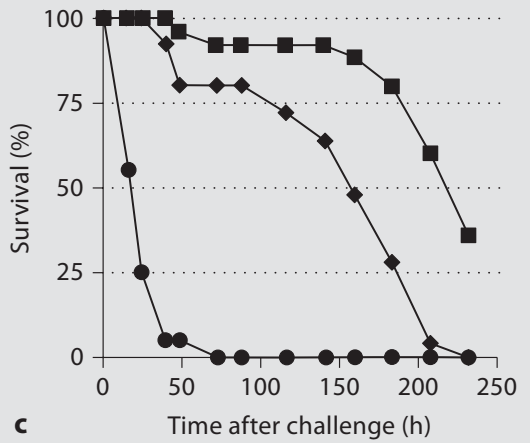

B. subtilis

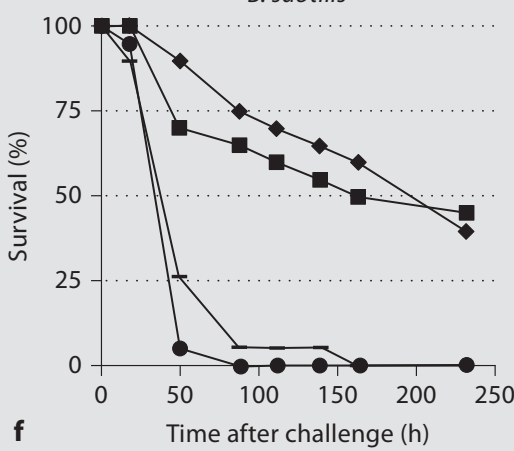

S. typhimurium

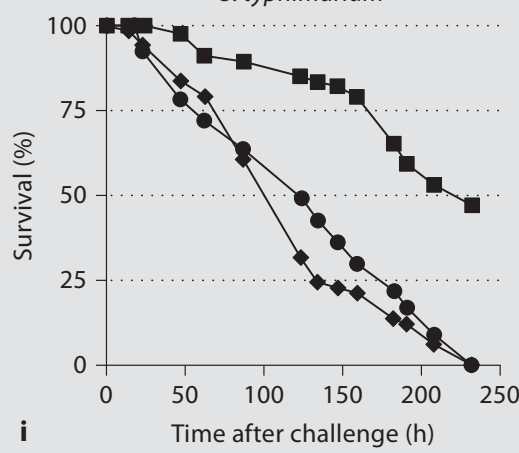

\ Parental wild type

- Phagoless

- Relish E20

- spzrm7

○ Conditional wild-type

$\times$ Conditional Phagoless 
This observation correlates with a reported role for circulating cells in the fat body response to injury in adults [25] and their production of clotting factor $[22,23]$. This observation suggests that adult phagocytes may also contribute to local responses to wounding, as observed in embryos $[36,37]$ and larvae $[38,39]$, probably by engulfing wound debris at the wound site and contributing to clot formation.

Compared with wild-type flies, Phago ${ }^{\text {less }}$ flies showed a late and mild increase in their susceptibility to systemic infections with the Gram-negative rods Ecc15 (fig. 3c) and E. coli (fig. 3d) or the Gram-positive coccus E. faecalis (fig. 3e). This is in sharp contrast with Rel and spz mutants, which showed an acute and strong susceptibility to infection with these Gram-negative rods or Gram-positive cocci, respectively (fig. 3c-e). Phago ${ }^{\text {less }}$ adults resisted as wild-type animals to infection by the Gram-positive bacillus $B$. subtilis (fig. 3f), the Gram-positive cocci $S$. saprophyticus (fig. 3g) and S. agalactiae (fig. $3 \mathrm{~h}$ ) or the Gram-negative rod E. cloacae (data not shown). These results contrast with the strong susceptibility of Toll and/or Imd pathway mutant flies (fig. $3 \mathrm{f}-\mathrm{h}$, data not shown). In contrast, Phago ${ }^{\text {less }}$ animals were severely affected, compared with wild-type animals, in their resistance to systemic infection by the Gram-negative rod S. typhimurium or the Gram-positive coccus S. aureus (fig. 3i, j). Of note, upon infection by those germs, Phago ${ }^{\text {less }}$ flies exhibited the same level of susceptibility than Toll and Imd pathway mutants, respectively.

To test if the observed susceptibility to bacterial infection was directly associated with phagocyte ablation, we generated conditional Phagoless individuals using an additional ubiquitously expressed transgene encoding a thermosensitive version of the GAL4 inhibitor, GAL80 [40]. When reared at permissive temperature $\left(18^{\circ} \mathrm{C}\right.$, GAL80 on, GAL4 system off) during the entire development, conditional Phago ${ }^{\text {less }}$ emerging adults had phagocytes and their developmental viability was similar to their wild-type siblings ( $95 \%, \mathrm{n}=55$; data not shown). We then cultured them at restrictive temperature $\left(29^{\circ} \mathrm{C}\right.$, GAL80 off, GAL4 system on) for 7 days. As observed with Phago $^{\text {less }}$ escapers, conditional Phago ${ }^{\text {less }}$ adults were depleted of phagocytes (data not shown) and showed an increased susceptibility to $S$. aureus infection (fig. 3k). These results demonstrate that the susceptibility observed is indeed associated with adult phagocyte depletion and indicate that adult phagocytes contribute to the host survival to several bacterial infections and are important to resist $S$. aureus and S. typhimurium systemic infections.

\section{The Humoral Antimicrobial Responses of Phago less} Animals Are Not Affected

Previous reports have suggested that the activation of Imd-mediated synthesis of antimicrobial peptides by the fat body of Drosophila larvae relies, at least partially, on the activity of haemocytes upon ingestion of the Gramnegative rod $E c c 15[26,27]$ or upon E. coli injection into the body cavity [28]. However, other reports suggest that phagocytes are not essential for this process $[41,42]$. We wondered if the activation of the Toll or Imd pathway relies on phagocytes at the adult stage. To address this question, we monitored the expression of specific immune genes regulated either by the Toll pathway (IM1), the Imd pathway (Diptericin) or both pathways (Defensin) upon $S$. aureus and $S$. typhimurium infections in Phago ${ }^{\text {less }}$ adults. As observed in wild-type flies, both IM1 and Defensin genes were significantly induced in Phago ${ }^{\text {less }}$ individuals upon S. aureus infection (fig. 4a, b). Similarly, both Diptericin and Defensin genes were strongly induced in Phago ${ }^{\text {less }}$ individuals as observed in wild-type animals upon $S$. typhimurium infection (fig. 4c, d). These results suggest that both Toll and Imd pathway activities upon infection with $S$. aureus and $S$. typhimurium are not affected by the absence of phagocytes. To further test this hypothesis, we assayed Diptericin and IM1 expression in Phago ${ }^{\text {less }}$ individuals upon injection of purified DAP-type PGN (DAP-PGN) from Gram-negative rods (E. coli) or purified Lys-type PGN (Lys-PGN) from Gram-positive cocci (E. faecalis) that are potent elicitors of the Imd and Toll pathways, respectively [32]. Interestingly, both Diptericin and IM1 genes were induced as in wild-type or even higher in Phagoless animals upon DAP-PGN and Lys-PGN injection, respectively (fig. 4e, f). Similar results were obtained when a mixture of E. coli and M. luteus bacteria was injected in wild-type and Phago ${ }^{\text {less }}$ adults (data not shown). Altogether, these results demonstrate that Imd and Toll pathway activities are normal in Phago ${ }^{\text {less }}$ animals and indicate that the increased susceptibility of Phago ${ }^{\text {less }}$ flies to S. aureus or S. typhimurium infections is not associated with a defect in the activation of Toll or Imd-dependent antimicrobial responses. Collectively, these results demonstrate that Drosophila adult humoral responses controlled by Toll and Imd signalling pathways are not significantly affected in the absence of phagocytes and support the notion that activation of the humoral arm of the Drosophila adult antimicrobial response does not rely on phagocyte activities. 
Fig. 4. The humoral antimicrobial response of Phago ${ }^{\text {less }}$ animals is not affected. a, b Quantitative RT-qPCR analysis of IMI (a) and Defensin (Def; b) induction after $S$. aureus septic injury in wild-type and Phago ${ }^{\text {less }}$ adults. c, d Quantitative RTqPCR analysis of Diptericin (Dpt; c) and Defensin (Def; d) induction after $S$. typhimurium septic injury in wild-type and Phago ${ }^{\text {less }}$ adults. e, f Quantitative RTqPCR analysis of Diptericin (Dpt; e) and IM1 (f) upon injection of purified DAPPGN from $E$. coli or purified Lys-PGN from E. faecalis in wild-type and Phago ${ }^{\text {less }}$ adults. $r p 49$ was used as the experimental expression standard. Relative $\Delta \mathrm{Ct}^{\text {gene }} /$ $\Delta \mathrm{Ct}^{r p 49}$ ratios of unchallenged wild-type controls were anchored in 1 to indicate fold induction. Graphs represent the mean and SD of relative ratios detected in 3 biological repetitions of a pool of 10 females. Genotypes: wild type: $h m l($ delta)-GAL4, UAS-eGFP/CyO-actin-GFP; Phago ${ }^{\text {less }}$ : hml(delta)-GAL4,UAS-eGFP/UAS-bax.
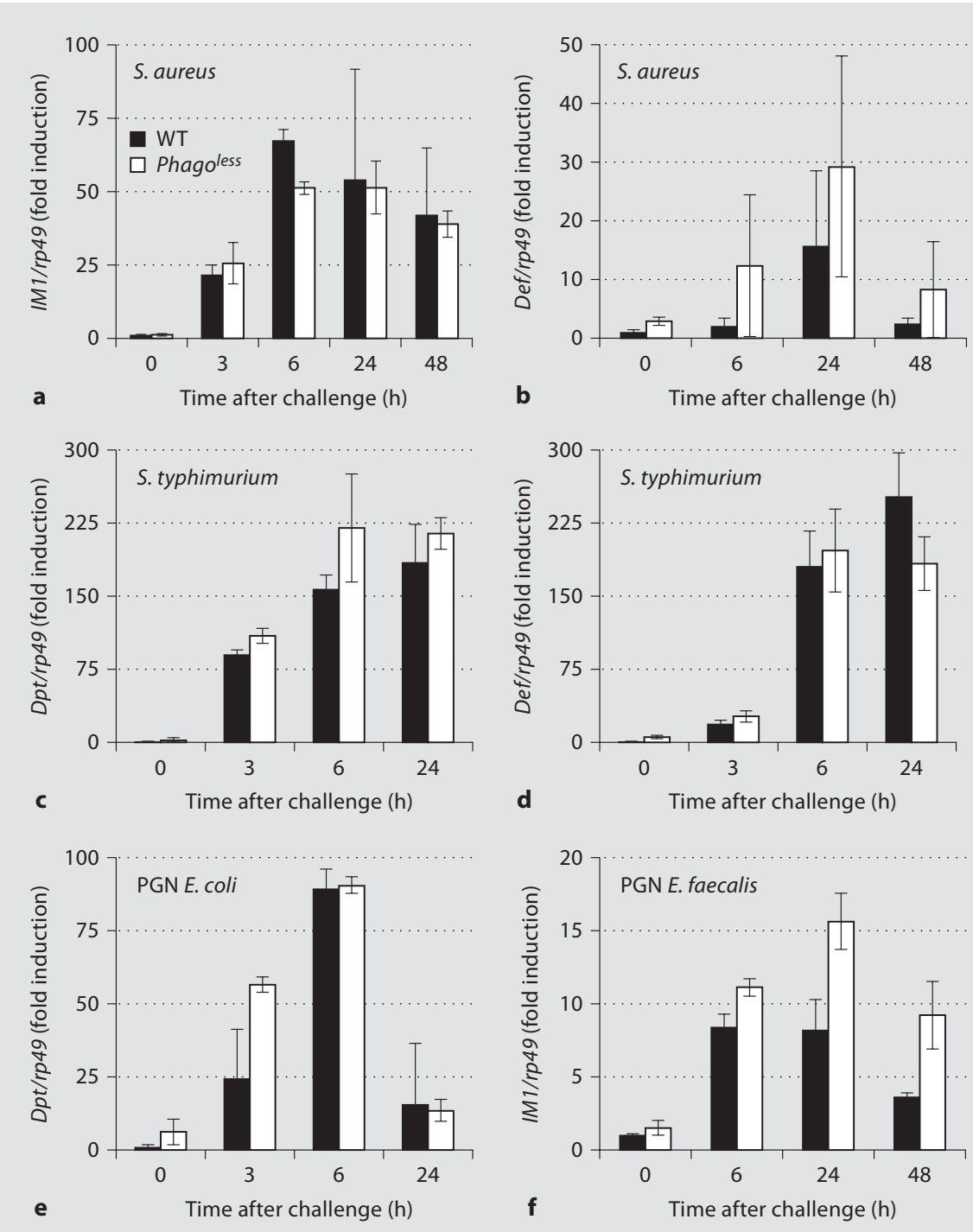

\section{Phagocytosis Contributes to Drosophila Resistance to Bacterial Infection}

In absence of any obvious defect in the activation of humoral immune responses, we wondered why Phago ${ }^{\text {less }}$ animals are less resistant than wild-type flies to $S$. aureus systemic infections. Of note, $S$. aureus is efficiently engulfed and digested by Drosophila phagocytes either ex vivo or in vivo [33]. Therefore, we hypothesized that phagocytosis may be an important resistance mechanism to $S$. aureus systemic infections in Drosophila. To test this hypothesis, we analysed the resistance to $S$. aureus infection of a Drosophila null mutant for Eater, a gene encoding a phagocytic receptor essential to mediate phagocytosis of S. aureus in vivo [33]. In addition, using the inducible in vivo RNAi technique, we selectively knocked down Eater expression in phagocytes using two independent UAS-Eater-RNAi transgenes driven by the $h \mathrm{ml}($ delta)-GAL4 driver and assayed the resistance of these Eater-RNAi flies to $S$. aureus infection. Figure 5 shows that both Eater null mutant flies and flies where Eater expression was selectively knocked down in phagocytes presented an increased susceptibility to $S$. aureus infection compared with wild-type flies. Of note, silencing of Eater expression led to the same increase in susceptibility as in Phago ${ }^{\text {less }}$ animals. Therefore, these results demonstrate that phagocytosis is a predominant defence mechanism employed by Drosophila phagocytes to fight $S$. aureus systemic infections and suggest that the 


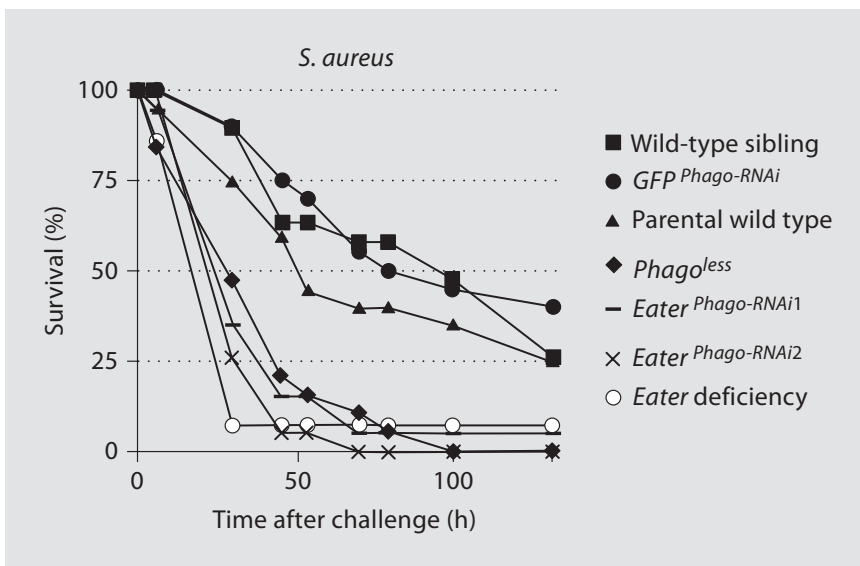

Fig. 5. Phagocytosis contributes to Drosophila resistance to systemic bacterial infection. Survival analysis of Phago ${ }^{\text {less }}$, wild-type siblings, wild-type parental control, GFP Phago-RNAi, Eater Phago-RNAil, Eater Phago-RNAi2 and Eater deficiency flies upon $S$. aureus septic injury. Genotypes: Phago ${ }^{\text {less }}$ : hml(delta)-GAL4, UAS-eGFP/UAS-bax; wild-type sibling: $h m l$ (delta)-GAL4,UASeGFP/CyO-actin-GFP; parental wild type: UAS-bax/CyO-actinGFP; GFP Phago-RNAi: $h m l$ (delta)-GAL4,UAS-eGFP/UAS-GFP-IR;

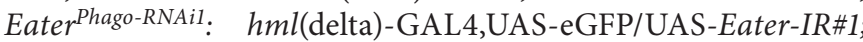
Eater $^{\text {Phago-RNAi2: }}$ hml(delta)-GAL4,UAS-eGFP/UAS-Eater-IR\#2; Eater deficiency: $D f(3 R) D 605 / D f(3 R) T l-I$.

enhanced susceptibility of Phago ${ }^{\text {less }}$ animals to S. aureus infection likely relies on the lack of this cellular defence mechanism.

\section{Discussion}

Based on their multiple developmental and immune functions, it has been inferred that Drosophila phagocytes play important roles during both development and immune responses. However, to our knowledge, no cell ablation experiments have ever been reported to confirm this. Here, we have challenged these ideas by generating Drosophila individuals lacking functional phagocytes. We now provide several lines of evidence supporting the essential requirement of haemocytes for embryonic development, the important contribution of larval phagocytes in host resistance to opportunistic infections during post-embryonic development and the critical role of adult phagocytes in the immune defence to several systemic bacterial infections.

Indeed, we have shown that specific ablation of Drosophila haemocytes leads to near complete lethality of the developing embryos indicating that haemocytes are es- sential for embryonic viability. In addition, we confirm that haemocytes contribute to CNS morphogenesis, in particular to VNC condensation. Interestingly, the abnormal CNS morphogenesis phenotypes of Haemo ${ }^{\text {less }}$ embryos are reminiscent of those observed in mutants that disrupt either haemocyte production (serpent) or migration (pvr) $[17,18]$. Similar phenotypes were also observed in embryos injected with croquemort dsRNA that impairs engulfment of apoptotic corpses by haemocytes [17] or when haemocytes express dsRNA of SPARC, a gene encoding a collagen binding extracellular matrix glycoprotein [19]. Collectively, our results and these observations demonstrate that haemocytes are essential for embryonic development most likely through their function of apoptotic corpse clearance and deposition of extracellular matrix components.

We also report that individuals depleted of phagocytes from early larval stages present a reduced developmental viability indicating that phagocytes ensure optimal survival during post-embryonic development. However, the emergence of viable and fertile Phago ${ }^{\text {less }}$ adults in proportion equivalent to wild-type siblings when reared in sterile conditions suggests that larval phagocytes are dispensable for normal post-embryonic development to proceed. Moreover, emerging germ-free Phago ${ }^{\text {less }}$ adults presented no obvious developmental delay or morphological defects apart from small melanotic masses found occasionally in the abdomen. Melanotic masses were also observed in animals reared under conventional condition and probably result from the encapsulation of incompletely eliminated larval tissues during metamorphosis. Indeed, it has been reported that Drosophila phagocytic cells participate in metamorphosis by ingesting doomed larval tissues, in particular muscle cells [11]. The fact that $P$ hago ${ }^{\text {less }}$ larvae and pupae were perfectly viable when raised in axenic conditions indicates that larval phagocytes ensure important immune functions during post-embryonic development rather than performing developmental functions as observed in embryos. This is supported by the previous observation by Matova and Anderson [42], who reported that double mutants lacking the Drosophila Rel proteins Dif and Dorsal are often infected by opportunistic microbes and die from infection during larval stages. Specific expression of either Dif or Dorsal in the larval blood cell lineage was sufficient to clear microbes and allow partial survival to the adult stage. In addition, Braun et al. [41] observed that domino and l(3) hem mutant, which are devoid of functional blood cells contain numerous live micro-organisms in their haemocoele in contrast to wild-type larvae. Hence, our 
results and these observations demonstrate that phagocytes significantly contribute to Drosophila larval viability in their standard laboratory environment, probably through clearance of opportunistic micro-organisms in the haemocoel.

Using adults depleted of phagocytes, we now demonstrate that these cells contribute to host resistance to systemic infections with specific bacteria. We observed that Phago ${ }^{\text {less }}$ individuals are as resistant as wild-type animals to B. subtilis, S. saprophyticus, S. agalactiae and E. cloacae infections while Toll or Imd pathway mutants present a striking enhanced susceptibility. This indicates that the cellular arm of Drosophila adult immune responses has either no role or is largely redundant against these germs. In contrast, Toll and Imd pathway-mediated immune responses play a key role. Nevertheless, the enhanced susceptibility of Phago ${ }^{\text {less }}$ animals to infections with $S$. aureus and S. typhimurium indicate that Drosophila phagocytes are critical players in the host resistance to these specific bacterial infections. The notion that phagocytes contribute to host resistance is further corroborated by the enhanced susceptibility of Phago ${ }^{\text {less }}$ flies to Ecc15, E. coli or E. faecalis infections compared with wild-type flies. In addition, the striking differences between the survival profiles of Phago ${ }^{\text {less }}$ animals and mutants affecting Toll and Imd pathways upon a large range of bacterial systemic infections indicate that the cellular and humoral antimicrobial defences have distinct and complementary functions in Drosophila adults. Recently, a report has suggested that the induction of the anti-bacterial peptide gene Defensin in the larval fat body during systemic infection requires blood cell contributions [28]. This points to a possible role of phagocytes in the activation of the antimicrobial peptide genes during systemic infection. This observation was controversial since previous studies did not reveal a role for phagocytes in this process at the larval stage $[41,42]$. Here, we clearly show that in adults, phagocytes are dispensable for the activation of Toll and Imd-dependent antimicrobial responses upon systemic infection. This contrasts with oral infection models, where larval blood cells contribute at least partially to Imd pathway activation in the fat body upon ingestion of the Gram-negative bacteria Erwinia carotovora $[26,27]$ while adult phagocytes actually dampen Imd pathway activation in the fat body upon ingestion of the entomopathogenic bacterium Serratia marcescens [43].

Our results also indicate that phagocytosis is probably the main mechanism by which phagocytes contribute to resistance to systemic bacterial infection. Indeed, Drosophila null mutants for Eater, a gene encoding a phago- cytic receptor essential to mediate phagocytosis of $S$. aureus in vivo [33], presented an increased susceptibility to S. aureus infection similar to Phago ${ }^{\text {less }}$ animals. This was also observed in flies where Eater expression was selectively knocked down by RNA interference in phagocytes. Interestingly, Toll and Imd pathway activities are not affected in Eater mutant flies upon systemic bacterial infection [33]. These observations demonstrate that phagocytosis contributes to resistance to systemic bacterial infection and acts as a complementary defence mechanism to antimicrobial humoral responses. This complementary role was suggested by a previous study that revealed the enhanced susceptibility to $E$. coli systemic infection of Imd mutant flies injected with polystyrene beads to saturate the phagocytic ability of adult phagocytes [44]. Finally, the importance of phagocytes upon systemic bacterial infection correlates with their critical role in host defence against oral infections by the entomopathogenic bacterium S. marcescens $[33,43]$.

Taken together, our observations indicate that the immune system of Drosophila adults relies on both the cellular and humoral defence mechanisms to resist systemic bacterial infections. However, the relative contribution of the cellular versus the humoral arm of its antimicrobial defence mechanisms appears to be highly dependent on the bacterial species. The contribution of phagocytes to host survival seems dependent on the specific virulence strategies employed by the infectious bacteria. Indeed, a clear contribution of phagocytes to host survival has so far only been revealed either when the humoral response was affected [44] or upon infection with pathogenic bacteria (S. marcescens, S. aureus, S. typhimurium) [this study and 33]. In contrast, the prevalence of Toll and/or Imd-mediated humoral responses for the host survival has been revealed upon all tested systemic bacterial infection, indicating a broader contribution of these humoral immune responses. Our study suggests that systemic infection by S. typhimurium or S. aureus are good infection models to study the molecular function of phagocytes in Drosophila.

In conclusion, we have now established a novel strategy to efficiently and selectively ablate phagocytes allowing the in vivo analysis of their contribution to specific biological processes in an invertebrate animal model, $D$. melanogaster. This completes previous phagocyte depletion strategies developed in vertebrates, which include suicide gene expression by macrophages in transgenic mice $[45,46]$ or the targeted disruption of the myeloid transcription factor pu.1 expression in mouse or zebrafish $[47,48]$. These strategies have been instrumental for 
the appreciation of phagocyte functions in vertebrates but remained limited given the partial ablation generated or the developmental lethality induced $[49,50]$. Using a selective and efficient phagocyte ablation strategy in Drosophila, we now provide a blueprint of phagocyte importance during both development and innate immune responses. Given the technical potential of the Drosophila model, our results pave the way to tissue-specific in vivo systematic genome-wide RNA interference screens to identify all genes important for phagocyte activities.

\section{Acknowledgments}

We thank Julien Royet for sharing unpublished data and his support to A.D., Isabelle Vallet-Gely for her critical reading of the manuscript, Bernard Mignotte, Bernard Mathey-Prevot, Katja Bruckner, Christine Kocks, the Bloomington stock center, the Vienna Drosophila RNAi Center and the National Institute of Genetics Fly Stock Centre for the fly lines, and Dominique MenginLecreulx for the gift of purified peptidoglycan preparations. A.D. is funded by a doctoral fellowship from the French Research Ministry. W.W.'s lab is funded by a Wellcome Trust Career Development Fellowship. This work was supported by the French National Research Agency (ANR-05-MIIM-016-01, to B.L.) and the Association pour la Recherche sur le Cancer (to B.L.).

\section{References}

1 Gordon S: The macrophage: past, present and future. Eur J Immunol 2007;37(suppl 1): S9-S17.

-2 Evans CJ, Hartenstein V, Banerjee U: Thicker than blood: conserved mechanisms in Drosophila and vertebrate hematopoiesis. Dev Cell 2003;5:673-690.

-3 Hartenstein V: Blood cells and blood cell development in the animal kingdom. Annu Rev Cell Dev Biol 2006;22:677-712.

$\checkmark 4$ Wood W, Jacinto A: Drosophila melanogaster embryonic haemocytes: masters of multitasking. Nat Rev Mol Cell Biol 2007;8:542551.

5 Stuart LM, Ezekowitz RA: Phagocytosis and comparative innate immunity: learning on the fly. Nat Rev Immunol 2008;8:131-141.

-6 Tepass U, Fessler LI, Aziz A, Hartenstein V: Embryonic origin of hemocytes and their relationship to cell death in Drosophila. Devel opment 1994;120:1829-1837.

7 Rehorn KP, Thelen H, Michelson AM, Reuter R: A molecular aspect of hematopoiesis and endoderm development common to vertebrates and Drosophila. Development 1996; 122:4023-4031.

$\checkmark 8$ Lebestky T, Chang T, Hartenstein V, Banerjee U: Specification of Drosophila hematopoietic lineage by conserved transcription factors. Science 2000;288:146-149.

$\checkmark 9$ Bataille L, Auge B, Ferjoux G, Haenlin M, Waltzer L: Resolving embryonic blood cell fate choice in Drosophila: interplay of GCM and RUNX factors. Development 2005;132: 4635-4644.

$\checkmark 10$ Holz A, Bossinger B, Strasser T, Janning W, Klapper R: The two origins of hemocytes in Drosophila. Development 2003;130:49554962.

11 Lanot R, Zachary D, Holder F, Meister M: Post-embryonic hematopoiesis in Drosophila. Dev Biol 2000;230:243-257.
12 Bidla G, Dushay MS, Theopold U: Crystal cell rupture after injury in Drosophila requires the JNK pathway, small GTPases and the TNF homolog Eiger. J Cell Sci 2007;120: 1209-1215.

13 Jung SH, Evans CJ, Uemura C, Banerjee U: The Drosophila lymph gland as a developmental model of hematopoiesis. Development 2005; 132:2521-2533.

14 Krzemien J, Dubois L, Makki R, Meister M, Vincent A, Crozatier M: Control of blood cell homeostasis in Drosophila larvae by the posterior signalling centre. Nature 2007;446: 325-328.

15 Mandal L, Martinez-Agosto JA, Evans CJ Hartenstein V, Banerjee U: A Hedgehog- and Antennapedia-dependent niche maintains Drosophila haematopoietic precursors. Nature 2007;446:320-324.

16 Lemaitre B, Hoffmann J: The host defense of Drosophila melanogaster. Annu Rev Immunol 2007;25:697-743.

17 Sears HC, Kennedy CJ, Garrity PA: Macrophage-mediated corpse engulfment is required for normal Drosophila CNS morphogenesis. Development 2003;130:3557-3565.

18 Olofsson B, Page DT: Condensation of the central nervous system in embryonic Drosophila is inhibited by blocking hemocyte migration or neural activity. Dev Biol 2005; 279:233-243.

19 Martinek N, Shahab J, Saathoff M, Ringuette M: Haemocyte-derived SPARC is required for collagen-IV-dependent stability of basal laminae in Drosophila embryos. J Cell Sci 2008;121:1671-1680.

20 Samakovlis C, Kimbrell D, Kylsten P, Engstrom A, Hultmark D: The immune response in Drosophila: pattern of cecropin expression and biological activity. EMBO J 1990;9: 2969-2976.
21 Reichhart J, Meister M, Dimarcq J, Zachary D, Hoffmann D, Ruiz C, Richards G, Hoffmann J: Insect immunity: developmental and inducible activity of the Drosophila diptericin promoter. EMBO J 1992;11:14691477.

22 Goto A, Kadowaki T, Kitagawa Y: Drosophila hemolectin gene is expressed in embryonic and larval hemocytes and its knock down causes bleeding defects. Dev Biol 2003;264: 582-591.

23 Korayem AM, Fabbri M, Takahashi K, Scherfer C, Lindgren M, Schmidt O, Ueda R, Dushay MS, Theopold U: A Drosophila salivary gland mucin is also expressed in immune tissues: evidence for a function in coagulation and the entrapment of bacteria. Insect Biochem Mol Biol 2004;34:1297-1304.

24 Pastor-Pareja JC, Wu M, Xu T: An innate immune response of blood cells to tumors and tissue damage in Drosophila. Dis Model Mech 2008;1:144-154.

-25 Agaisse H, Petersen UM, Boutros M, MatheyPrevot B, Perrimon N: Signaling role of hemocytes in Drosophila JAK/STAT-dependent response to septic injury. Dev Cell 2003; 5:441-450.

26 Basset A, Khush R, Braun A, Gardan L, Boccard F, Hoffmann J, Lemaitre B: The phytopathogenic bacteria, Erwinia carotovora, infects Drosophila and activates an immune response. Proc Natl Acad Sci USA 2000;97: 3376-3381.

27 Dijkers PF, O'Farrell PH: Drosophila calcineurin promotes induction of innate immune responses. Curr Biol 2007;17:20872093

28 Brennan CA, Delaney JR, Schneider DS, Anderson KV: Psidin is required in Drosophila blood cells for both phagocytic degradation and immune activation of the fat body. Curr Biol 2007;17:67-72.

29 Gaumer S, Guenal I, Brun S, Theodore L, Mignotte B: Bcl-2 and Bax mammalian regulators of apoptosis are functional in Drosophila. Cell Death Diff 2000;7:804-814. 
30 Bruckner K, Kockel L, Duchek P, Luque CM, Rorth P, Perrimon N: The PDGF/VEGF receptor controls blood cell survival in Drosophila. Dev Cell 2004;7:73-84.

-31 Sinenko SA, Mathey-Prevot B: Increased expression of Drosophila tetraspanin, Tsp68c, suppresses the abnormal proliferation of ytrdeficient and Ras/Raf-activated hemocytes. Oncogene 2004;23:9120-9128.

\$2 Leulier F, Parquet C, Pili-Floury S, Ryu JH, Caroff M, Lee WJ, Mengin-Lecreulx D, Lemaitre B: The Drosophila immune system detects bacteria through specific peptidoglycan recognition. Nat Immunol 2003;4:478484.

$\checkmark 33$ Kocks C, Cho JH, Nehme N, Ulvila J, Pearson AM, Meister M, Strom C, Conto SL, Hetru C, Stuart LM, Stehle T, Hoffmann JA, Reichhart JM, Ferrandon D, Ramet M, Ezekowitz RA: Eater, a transmembrane protein mediating phagocytosis of bacterial pathogens in Drosophila. Cell 2005;123:335-346.

34 Brand AH, Perrimon N: Targeted gene expression as a means of altering cell fates and generating dominant phenotypes. Development 1993;118:401-415.

-35 Adams JM, Cory S: The Bcl-2 protein family: arbiters of cell survival. Science 1998;281: 1322-1326.

-36 Stramer B, Wood W, Galko MJ, Redd MJ, Jacinto A, Parkhurst SM, Martin P: Live imaging of wound inflammation in Drosophila embryos reveals key roles for small GTPases during in vivo cell migration. J Cell Biol 2005; 168:567-573.
37 Wood W, Faria C, Jacinto A: Distinct mechanisms regulate hemocyte chemotaxis during development and wound healing in Drosophila melanogaster. J Cell Biol 2006;173: 405-416.

38 Galko MJ, Krasnow MA: Cellular and genetic analysis of wound healing in Drosophila larvae. PLoS Biol 2004;2:E239.

39 Babcock DT, Brock AR, Fish GS, Wang Y, Perrin L, Krasnow MA, Galko MJ: Circulating blood cells function as a surveillance system for damaged tissue in Drosophila larvae. Proc Natl Acad Sci USA 2008;105:1001710022.

40 McGuire SE, Mao Z, Davis RL: Spatiotemporal gene expression targeting with the target and gene-switch systems in Drosophila. Sci STKE 2004;2004:pl6.

41 Braun A, Hoffmann JA, Meister M: Analysis of the Drosophila host defense in domino mutant larvae, which are devoid of hemocytes. Proc Natl Acad Sci USA 1998;95: 14337-14342.

42 Matova N, Anderson KV: Rel/NF-kappaB double mutants reveal that cellular immunity is central to Drosophila host defense. Proc Natl Acad Sci USA 2006;103:1642416429.

43 Nehme NT, Liegeois S, Kele B, Giammarinaro P, Pradel E, Hoffmann JA, Ewbank JJ, Ferrandon D: A model of bacterial intestinal infections in Drosophila melanogaster. PLoS Pathog 2007;3:e173.
44 Elrod-Erickson M, Mishra S, Schneider D: Interactions between the cellular and humoral immune responses in Drosophila. Curr Biol 2000;10:781-784.

-45 Burnett SH, Kershen EJ, Zhang J, Zeng L, Straley SC, Kaplan AM, Cohen DA: Conditional macrophage ablation in transgenic mice expressing a Fas-based suicide gene. J Leukoc Biol 2004;75:612-623.

46 Cailhier JF, Partolina M, Vuthoori S, Wu S, Ko K, Watson S, Savill J, Hughes J, Lang RA: Conditional macrophage ablation demonstrates that resident macrophages initiate acute peritoneal inflammation. J Immunol 2005;174:2336-2342.

47 McKercher SR, Torbett BE, Anderson KL, Henkel GW, Vestal DJ, Baribault H, Klemsz M, Feeney AJ, Wu GE, Paige CJ, Maki RA: Targeted disruption of the PU.1 gene results in multiple hematopoietic abnormalities. EMBO J 1996;15:5647-5658.

-48 Clay H, Davis JM, Beery D, Huttenlocher A, Lyons SE, Ramakrishnan L: Dichotomous role of the macrophage in early Mycobacterium marinum infection of the zebrafish. Cell Host Microbe 2007;2:29-39.

49 Shepard JL, Zon LI: Developmental derivation of embryonic and adult macrophages. Curr Opin Hematol 2000;7:3-8.

50 Rhodes J, Hagen A, Hsu K, Deng M, Liu TX, Look AT, Kanki JP: Interplay of pu.1 and gatal determines myelo-erythroid progenitor cell fate in zebrafish. Dev Cell 2005;8: 97-108. 